\title{
CAPITAL SOCIAL Y DESARROLLO EN ZONAS RURALES Un análisis de los programas Leader II y Proder en Andalucía
}

\author{
FERNANDO E. GARRIDO FERNÁNDEZ y EDUARDO MOYANO ESTRADA \\ IESA-CSIC, Córdoba
}

\section{PALABRAS CLAVE ADICIONALES}

Desarrollo local, Acción colectiva, Sociedad rural, España.

\section{ADDITIONAL KEYWORDS}

Local Development, Collective Action, Rural Society, Spain.

RESUMEN. En este artículo se analizan los programas Leader y Proder de desarrollo en zonas rurales utilizando la perspectiva del "capital social". En primer lugar, se presentan los elementos fundamentales de la noción de "capital social", ofreciendo un breve recorrido por el pensamiento sociológico de modo que podamos encontrar sus fuentes intelectuales. En segundo lugar, se presenta el marco teórico elaborado por $\mathrm{M}$. Woolcock, como un modelo-síntesis para analizar los problemas que se plantean en las políticas de desarrollo. Finalmente, se exponen los resultados de un análisis experimental realizado en el marco de la evaluación de la iniciativa europea de desarrollo rural Leader II y del programa Proder en Andalucía.

ABSTRACT. In this article the Leader and Proder schemes for rural areas are analysed from the social capital approach. Firstly, their authors review the main dimensions of the notion of social capital and analyse its theoretical sources in the social sciences. Secondly, they show the framework elaborated by Michael Woolcock as a tool to analyse the dynamics of development in local areas. Finally, they offer the results of an empirical research on the implementation process of the Leader and Proder schemes in the Spanish region of Andalusia using the capital social approach.

E-mail: fgarrido@iesaa.csic.es emoyano@iesaa.csic.es

Revista Internacional de Sociología (RIS)

Tercera Época, $n^{\circ} 33$, Septiembre-Diciembre, 2002, pp. 67-96. 
RIS

REVISTA INTERNACIONAL DE SOCIOLOCIA

№ 33, Septiembre-Diciembre, 2002

FERNANDO E. GARRIDO FERNÁNDEZ y EDUARDO MOYANO ESTRADA

\section{INTRODUCCIÓN}

En los estudios sobre el desarrollo cada vez resulta más frecuente encontrar diferencias significativas en materia de bienestar entre sociedades situadas en un mismo ámbito geográfico y dotadas de similares recursos económicos y humanos. En efecto, es bastante común encontrar a pocos kilómetros de distancia sociedades que no han sido capaces de alcanzar unas cotas mínimas de bienestar y de organizar adecuadamente sus recursos, junto a otras que han sabido aprovecharlos y están mejor articuladas para llevar a cabo proyectos individuales o colectivos en beneficio de la comunidad. Puede que incluso ambas sociedades hayan sido objeto preferente de programas similares de desarrollo. Pero la realidad es que mientras, en las primeras, esos programas no han tenido el éxito esperado, en las segundas sus efectos han superado con creces lo previsto en los estudiơs exante realizados por los expertos. Estas últimas se convierten además en centros de atracción de nuevas inversiones, al comprobar los posibles inversores que ellas ofrecen un dinamismo que no existe en las otras y que son garantía para la buena realización de los proyectos que se ponen en marcha. En estas comunidades, las instituciones funcionan con eficiencia, sus recursos humanos son cualificados, existe seguridad jurídica para la iniciativa privada, la ciudadanía confía en sus gobernantes y éstos en las capacidades de su población; hay, en definitiva, confianza entre los ciudadanos para emprender proyectos colectivos.

Estos contrastes en los resultados de los programas de desarrollo han originado una ingente cantidad de estudios intentando explicar sus causas. Después de la hegemonía, primero, de los estudios basados en los enfoques de la modernización, y, más tarde, de los que explicaban el problema del subdesarrollo a partir de las teorías de la dependencia, o de los que insistían en las recetas liberalizadoras como la mejor vía para impulsar las iniciativas locales, más recientemente se han venido realizando algunos trabajos que han enfatizado la importancia de aspectos como la confianza, los flujos de información o las normas de reciprocidad existentes en una comunidad; es decir, aspectos que componen el denominado enfoque del capital social. El Banco Mundial, en su Informe de 1997, ya señalaba cómo algunos estudios referidos al continente africano estaban demostrando el potencial impacto de estos otros factores no económicos sobre el desarrollo de las comunidades locales, así como sobre la provisión de bienes públicos y la eficacia de las agencias estatales (pág. 114 del Informe del Banco Mundial, citado por J. Fedderke et al., 1999). Como señala M. Woolcock (1998), estos últimos estudios han recuperado, redefiniéndola, la vieja noción de capital social, ofreciendo la posibilidad de establecer puentes entre disciplinas hasta ahora separadas en el análisis de los problemas que afectan a los países en vías de desarrollo (economía, ciencia política, sociología, antropología, psicología social, historia....).

No hay que ir a los países en vías de desarrollo para encontrar estos contrastes. En algunas regiones españolas donde se están abordando programas de desarrollo 
local/rural, como son la iniciativa europea Leader y el programa Proder, también podemos encontrar estas diferencias entre comunidades pertenecientes a un mismo ámbito geográfico y dotadas de recursos productivos muy similares. Hay ya suficiente información para proceder a un análisis exhaustivo del impacto de estos programas, cuyos resultados pueden verse en las evaluaciones a que se han sometido tales programas. No es el objetivo de este artículo exponer los resultados de esas evaluaciones, sino interpretarlos desde la mencionada perspectiva del capital social. Para ello, utilizaremos los resultados de una aplicación experimental de la noción de capital social a la evaluación de la iniciativa Leader II y del programa Proder en Andalucía realizada en el año 2002'.

En este artículo presentaremos, en primer lugar, el enfoque del capital social, ofreciendo un breve recorrido por el pensamiento sociológico de modo que podamos encontrar sus fuentes intelectuales. En segundo lugar, se presenta el marco teórico elaborado por M. Woolcock, como un modelo-síntesis para analizar los problemas que se plantean en las políticas de desarrollo. Finalmente, se exponen los resultados del mencionado análisis experimental realizado en el marco de la evaluación de la iniciativa Leader II y del programa Proder en Andalucía.

\section{EL ENFOQUE DEL CAPITAL SOCIAL EN LOS ESTUDIOS SOBRE DESARROLLO}

El enfoque del capital social utiliza un concepto que no es nuevo, sino que hunde sus raíces en los primeros trabajos de las ciencias sociales ${ }^{2}$. Precisamente por no ser un concepto de nuevo cuño, su utilización presenta el problema de que los autores que lo han recuperado en sus estudios sobre el desarrollo, lo han hecho sin prestar mucha atención ni a su historia intelectual, ni a su status ontológico, sino simplemente enfatizando aquellas dimensiones del mismo que les son más útiles para sus particulares propósitos investigadores. Por ello, nos encontramos

\footnotetext{
${ }^{1}$ La evaluación fue realizada por la empresa pública DAP de la Consejería de Agricultura de la Junta de Andalucía; en dicha evaluación colaboró el IESA-CSIC analizando las opiniones de la población respecto a la aplicación de dichos programas y al grado de implicación de las distintas instituciones.

${ }^{2} \mathrm{La}$ idea de que es necesario que existan determinadas normas de cooperación para guiar las transacciones mercantiles, puede ya encontrarse en D. Hume y E. Burke, si bien de un modo ambivalente. Por su parte, A. Smith ya señaló en su Teoria de los Sentimientos Morales que el mercado necesitaba de ciertas instituciones y sensibilidades de carácter moral para funcionar eficientemente, dado que su capacidad de autorregulación es limitada. Los trabajos de los primeros sociólogos franceses del siglo XIX y, más tarde, los realizados en el marco de las tradiciones marxista, durkheimiana y weberiana de la sociología clásica, enfatizaron el papel de las instituciones y las normas sociales, planteando muchas de las ideas que ahora se incluyen dentro de la noción de capital social.
} 
RIS

con un concepto polisémico de capital social, que no presenta una acepción única en la comunidad científica, sino una gama de significados según la dimensión enfatizada.

\section{Antecedentes}

Al final de los años 80 , sociólogos, politólogos y algunos economistas —que trabajaban en el campo de la "nueva sociología económica" y que intentaban situarse en una posición intermedia combinando enfoques macro y microsociológicos para explicar el comportamiento económico de los individuos (Swedberg, 1991, y Swedberg y Smelser, 1994) — consideraron que el capital físico (tierra y capital) y el capital humano (nivel de estudios) eran insuficientes para explicar las diferencias en los procesos de desarrollo entre comunidades, ya que enteñdían que había otros factores que intervenían en tales procesos, pero que no eran incluidos en esos dos tipos de capital. Por eso añadieron un tercer factor, al que llamaron capital social, recuperando un término que estaba siendo utilizado en el campo de la sociología desde final de los años 60 en áreas diversas de investigación ${ }^{3}$. En esta nueva forma de capital incluían determinados tipos de normas, valores y creencias (como la confianza entre vecinos, el respeto a la palabra dada o la credibilidad de las instituciones), así como de redes sociales (de ahí que también suela denominarse "capital relacional"), que, según esta perspectiva teórica, son importantes para el desarrollo porque favorecen la realización de acciones colectivas en beneficio de la propia comunidad y repercuten en el buen aprovechamiento de los otros dos tipos de capital (Fedderke et al., 1999).

\section{El modelo bidimensional del capital social}

El avance más significativo en los estudios sobre desarrollo utilizando el enfoque del capital social se produjo entre el final de los 80 y toda la década de los

\footnotetext{
${ }^{3}$ Primero, fue J. Jacobs (1961), luego P. Bourdieu y J.C. Passeron (1970) y, más tarde, G. Loury (1977 a y b) los que utilizaron este concepto. Quienes mejor lo han utilizado en investigaciones empíricas han sido el propio G. Loury (1977 a y b) -que lo utilizó en su estudio sobre adolescencia y relaciones familiares-, J. Coleman (1988 y 1990) -en su estudio sobre el abandono de los centros escolares por los jóvenes estadounidenses-, R. Burt (1992), R. Putnam -en su estudio sobre Italia (1993) y más recientemente en estudios sobre la sociedad norteamericana, tomando como base las relaciones sociales que se desarrollan en las boleras (1995)-y A. Portes y J. Sensenbrenner (1993) -en sus estudios sobre el empresariado en grupos étnicos. En España, la escasa literatura que todavía existe sobre capital social está siendo acelerada en los últimos años con aportaciones como las de J.R. Montero y M. Torcal (2000), C. Boix y N. Posner (2000) y F. Herreros y A. de Francisco (2001). A ello habría que unir la revitalización de la tradición republicana, que enfatiza la importancia de los valores cívicos y el capital social (Herreros, 2001). Sobre capital social y confianza puede verse la tesis doctoral de F. Herreros, así como algunos de sus trabajos de investigación (2002a y b).
} 
90. Tal avance vino de la mano de dos perspectivas diferentes dentro de lo que se denomina "nueva sociología del desarrollo", a saber: en el nivel micro, de la perspectiva de los estudios sobre el empresariado en grupos étnicos, asociados a los trabajos de I. Light, A. Portes, J. Sensenbrenner y R. Waldinger; y en el nivel macro, de la perspectiva de los estudios institucionalistas sobre las relaciones Estado-sociedad, asociados a los trabajos de R. Bates, A. Amsden, P. Evans y R. Wade. Estas perspectivas han realizado sus investigaciones con escasa conexión entre sí, a pesar de que presentan ideas que podrían ser utilizadas en una síntesis de gran fertilidad para los estudios del desarrollo.

Ambas perspectivas coincidían en reafirmar la centralidad de dos ideas que se incorporarán más tarde, como dimensiones complementarias, al concepto de capital social. La primera es la idea de embeddedness (que en español podría traducirse como enraizamiento) y la segunda, la de autonomy (autonomía) ${ }^{4}$.

A final de los años 80, la idea del embeddedness fue incorporada en las investigaciones sobre el desarrollo, tanto en las centradas en el nivel macro, como en el micro. Tres conclusiones surgieron de esos estudios. La primera es la de que todas las formas de intercambio económico están enraizadas (embedded) en relaciones sociales; de ahí que muchas instituciones económicas sólo pueden explicarse por las relaciones sociales en que están insertas. La segunda conclusión es la de que el proceso de enraizamiento (embeddedness) se produce empíricamente de distintas formas: como lazos sociales, como prácticas culturales, como estructuras políticas..., todas ellas con efectos diversos en la conformación de las oportunidades y constricciones a las que las comunidades se enfrentan cuando emprenden proyectos de desarrollo. La tercera es la de que los beneficios que se obtienen de ese proceso de enraizamiento (embeddedness) en una comunidad concreta van siempre acompañados de costes, y que el cálculo de esos beneficios y costes cambia conforme el proceso de desarrollo avanza, de tal modo que lo que en una fase inicial puede ser catalogado como beneficio, en una fase más avanzada del desarrollo puede haberse convertido en un coste para la continuidad de dicho proceso. Por ejemplo, en una primera fase, la existencia de un alto grado de integración social y de una elevada densidad asociativa en una comunidad (ejemplos ambos de embeddedness) puede ser un factor positivo para desencadenar el proceso de desarrollo, al facilitar la ayuda mutua y la cooperación entre los individuos; pero una vez que dicho proceso está iniciado, ese stock de capital social en forma de integración en grupos primarios o de redes asociativas puede convertirse en una

\footnotetext{
${ }^{4}$ La idea de embeddedness deriva de K. Polanyi -que la desarrolló en su ya clásica obra The Great Transformation (1957)-, pero fue introducida en la sociología contemporánea por M. Granovetter (1985) al señalar, frente a la tesis neoclásica de la maximización de utilidades, que toda acción económica está enraizada (embedded) en relaciones sociales, y que son estas relaciones, concretadas en estructuras y redes personales, las que deben ser objeto de análisis.
} 
pesada carga de control social sobre los individuos o en un vehículo de nepotismo o corrupción y, en consecuencia, ser una traba para que dicha comunidad dé un salto cualitativo en su desarrollo.

Con el fin de establecer si el proceso de enraizamiento (embeddedness) en una situación dada provoca costes o beneficios para el desarrollo, algunos analistas comenzaron a sugerir que era necesario complementar esa dimensión del capital social con la de "autonomía" (autonomy). Esta segunda dimensión hace alusión al grado en que los miembros de una comunidad tienen autonomía para acceder a grupos o áreas de interés situados fuera de su propio grupo de pertenencia (familia, amigos, clanes...).

Las aportaciones de estos estudios sobre el desarrollo permitían concluir, por tanto, que, para convertir el capital social en un factor positivo del desarrollo de una comunidad, sería necesario que las relaciones sociales entre sus miembros estén impregnadas de esas dos dimensiones: embeddedness - enraizamiento en la propia comunidad - y autonomy - capacidad de los individuos para relacionarse con grupos más amplios 5 . No obstante, tales estudios también mostraban que, en la realidad empírica, ambas dimensiones se manifiestan de diversas formas, teniendo cada una de ellas efectos diferentes sobre las dinámicas de desarrollo, efectos que deben ser objeto de análisis en cada caso ${ }^{6}$. Por ello, hasta el final de los años 80 , la búsqueda de la combinación óptima de esas dos dimensiones del capital social (embeddedness y autonomy) se convirtió en el elemento funda-

${ }^{5}$ Fedderke et al. (1999) utilizan los conceptos de transparency (transparencia)-como equivalente al de embeddedness - y rationalization (racionalización) -como equivalente al de autonomy-para analizar los procesos de desarrollo económico, procesos en los cuales la principal función del capital social es reducir los costes de transacción. Para Fedderke et al., cuyo trabajo se centra en las implicaciones del capital social para el desarrollo económico, la "transparencia" sería aquel aspecto del capital social que, al reducir los costes de transacción, facilita la distribución de información en cantidad y calidad entre los individuos de una comunidad, de modo que aumenta la certidumbre de éstos en sus relaciones económicas intracomunitarias; la "racionalización" sería aquel otro aspecto del capital social que, al aumentar el grado de formalización de las normas y valores de una comunidad en lenguajes más universales y menos particularistas, favorece las relaciones de sus miembros con individuos de otras comunidades para emprender proyectos conjuntos de desarrollo.

${ }^{6}$ Centrándonos, por ejemplo, en la realidad de las zonas rurales, y más concretamente en organizaciones como las cooperativas agrarias o cualquier otra forma de economía social, la dimensión de embeddedness -es decir, la identificación de sus miembros con el correspondiente proyecto empresarial- es una condición necesaria por sus efectos positivos en una primera fase, pero no suficiente para hacer que el proceso de desarrollo sea sostenible en el largo plazo; es en esta fase cuando se hace necesario que tales empresas cooperativas tengan posibilidad de, y capacidad para, establecer relaciones autónomas con actores económicos externos - para emprender proyectos cooperativos de segundo grado, por ejemplo-, condición que no está garantizada con la existencia de un alto grado de embeddedness entre sus socios. 
mental del marco teórico que la nueva sociología del desarrollo utilizaba para analizar los procesos de desarrollo local/rural. Se pensaba que, encontrando para cada realidad empírica la combinación óptima de esas dos dimensiones, podrían resolverse algunos de los llamados dilemas estáticos de la acción colectiva-el problema de explicar por qué la gente coopera en ausencia de mecanismos de carácter obligatorio- que han ocupado a los investigadores sociales desde el comienzo de la sociología como disciplina científica.

Hacia mediados de los años 90, sociólogos que trabajaban en el campo del empresariado en grupos étnicos y del neoinstitucionalismo advirtieron sobre la dificultad de encontrar una combinación óptima de ambas dimensiones del capital social (embeddedness y autonomy), debido a que se pueden manifestar de modo diferente en los niveles micro y macro de los procesos de desarrollo. De acuerdo con esa argumentación, el sentido de las nociones de embeddedness y autonomy no es el mismo en los niveles micro y macro. Así, por ejemplo, mientras que en el nivel micro la noción de embeddedness se refiere a los lazos intracomunitarios que se establecen entre los individuos en una comunidad, en el nivel macro dicha dimensión se refiere al grado de interacción entre el Estado y la sociedad civil. Por su parte, la dimensión de autonomy se refiere, en el nivel micro, a las redes extracomunitarias - es decir, las redes que establecen los miembros de la comunidad con los de otras comunidades distintas de la suya-, mientras que, en el nivel macro, se refiere a la capacidad (eficiencia) y credibilidad de las instituciones encargadas de gestionar los asuntos públicos en una comunidad. De ahí se deduce que la noción de capital social pueda presentarse en la práctica de diversas formas, según cómo se combinen sus dos dimensiones (embeddedness y autonomy) en los niveles macro y micro de los procesos de desarrollo. De la combinación de esas dos dimensiones, y a la luz de determinadas experiencias, algunos estudios empíricos han señalado que altos niveles de capital social - por ejemplo, en el nivel micro, un fuerte enraizamiento de los individuos en sus grupos primarios, y en el nivel macro, una elevada dependencia de las asociaciones de la sociedad civil respecto de las instituciones públicas - pueden ser positivos en la medida en que dan a los individuos y asociaciones de una comunidad acceso a recursos que de otra forma no le serían fácilmente accesibles, pero también han señalado esos mismos estudios que pueden tener efectos negativos para el desarrollo si restringen las posibilidades de autonomía de los agentes sociales o si favorecen el free-ridding (gorroneo) de los individuos sobre los recursos de la comunidad.

Sin embargo, tal modelo, basado en la combinación de las dimensiones de embeddedness y autonomy, comenzó a encontrar problemas cuando se intentaba aplicar al análisis dinámico de los procesos de desarrollo; es decir, cuando lo que se quería analizar no era sólo las condiciones que permitieran iniciar con éxito la fase de implementación de tales procesos, sino también conocer los factores que pueden condicionar su viabilidad y sostenibilidad en el medio y largo plazo. 
RIS

REVISTA INTERNACIONAL DE SOCIOLOGIA

№ 33, Septiembre-Diciembre, 2002

FERNANDO E. GARRIDO FERNÁNDEZ y EDUARDO MOYANO ESTRADA

Con objeto de superar las limitaciones del modelo elaborado por la perspectiva del empresariado en grupos étnicos y por el enfoque neoinstitucionalista, $M$. Woolcock (1998) propone un modelo-síntesis al que dedicaremos el próximo apartado, al ser el modelo que hemos tomado como base para el análisis empírico de los programas Leader II y Proder en Andalucía.

\section{El modelo ampliado de Woolcock}

De acuerdo con $\mathrm{M}$. Woolcock, para que la noción de capital social mantenga su status como concepto significativamente importante en los estudios sobre el desarrollo, debe ser ampliada. No basta con entender el capital social como un recurso que explica la superación de los dilemas estáticos de la acción colectiva en una comunidad - los problemas de la cooperación entre los individuos en proyectos de tipo colectivo-, sino que - y ésa es la gran aportación de M. Woolcock - debe ser ampliado incorporando otras dimensiones que permitan resolver los llamados dilemas dinámicos del desarrollo, es decir, los que garantizan la continuidad de las dinámicas iniciadas y el éxito de las mismas a medio y largo plazo en una comunidad.

M. Woolcock considera necesario definir con más detalle las dimensiones de embeddedness y autonomy y analizar mejor cómo ambas se manifiestan en los niveles macro y micro. En el nivel micro, amplía la dimensión de embeddedness (enraizamiento) - que en el modelo anterior se refería sobre todo a los lazos sociales intracomunitarios, es decir, a las relaciones de los individuos con los de su propio grupo de referencia-y la sustituye por la noción de integration (integración) - que incluye también las relaciones con otros miembros de la cỏmunidad-; la dimensión de autonomy (autonomía) — que hacía referencia a la participación de los individuos en redes extracomunitarias- es sustituida por la de linkage (conexión) - incluyendo además la implicación de los individuos con las instituciones de la sociedad civil existentes en su comunidad.

En el nivel macro, la dimensión de embeddedness - referida en el primer modelo a las relaciones Estado/sociedad civil- es sustituida ahora por la de institutional synergy (sinergia entre instituciones) -incluyendo también la cooperación entre instituciones tanto públicas como privadas-; la dimensión de autonomy -referida en el primer modelo a la capacidad, competencia y credibilidad de las instituciones políticas locales-es ampliada y sustituida por la de organizational eficiency (eficiencia organizacional) 7 - que incluye, además, la eficiencia de la burocracia administrativa tanto en las instituciones públicas locales, como en las

\footnotetext{
${ }^{7}$ En el modelo de Woolcock esta dimensión es denominada organizational integrity. En este trabajo la denominamos eficiencia organizacional para evitar confusión con el término integration.
} 
organizaciones privadas. Estas cuatro dimensiones amplían el concepto de capital social y lo hacen más útil para analizar las dinámicas de desarrollo, combinando los niveles macro y micro (Figura $\mathrm{n}^{\circ} 1$ ).

Así, de acuerdo con los valores (altos o bajos) que, en los niveles micro y macro, pueden tomar esas cuatro dimensiones del capital social (integration, linkage, institutional synergy, organizational eficiency), nos encontramos con un amplio rango de hasta dieciséis situaciones a lo largo de un continuum en uno de cuyos extremos estaría la situación menos favorable para el desarrollo (denominada "individualismo anárquico" en el modelo de Woolcock) - en la que las cuatro dimensiones del capital social toman valores bajos-y en el otro la más favorable (denominada "autonomía positiva") — en la que tales dimensiones toman valores altos.

Figura $n^{\circ} 1$.

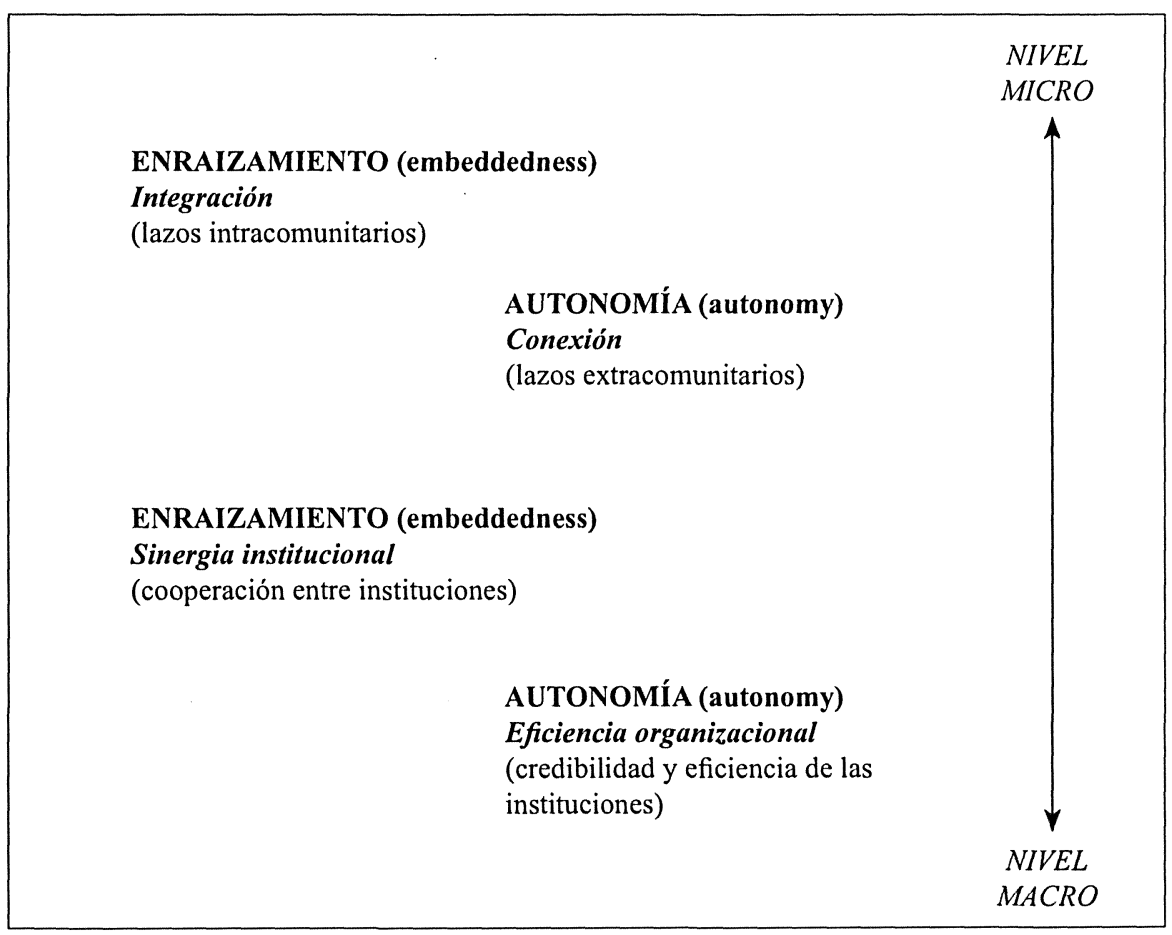

Fuente: Elaboración propia a partir de ilustraciones de Woolcock (1998). 


\section{APLICACIÓN AL ESTUDIO DE LOS PROGRAMAS LEADER II Y PRODER EN ANDALUCÍA}

La iniciativa europea Leader es una iniciativa de la Comisión Europea destinada a promover el desarrollo en las zonas rurales de la Unión Europea. Desde su aprobación en 1991, se han puesto en marcha tres de ellas: Leader I (1991-1994), Leader II (1995-2001) y Leader Plus (vigente desde el año 2002). Paralelamente, se ha venido aplicando en el territorio español un programa de desarrollo, denominado Proder, similar y complementario a aquél —concretamente, en las zonas no afectadas por las iniciativas Leader-, cuya diferencia estriba en que este último no es una iniciativa de la Comisión Europea, sino un programa operativo dentro del Programa de Desarrollo Regional presentado por el Gobierno español a las instituciones de la UE y aprobado por éstas con cargo a los Fondos Estructurales. A los efectos del tema tratado en este artículo nos interesa señalar que, independientemente de las diferencias entre ambos, los programas Leader y Proder responden al objetivo general de promover la movilización de los recursos endógenos en el territorio rural y la participación de los agentes locales, inspirándose en una concepción del desarrollo basada en los siguientes elementos:

- el enfoque territorial: se subraya con ello un proceso de desarrollo local que se basa en los recursos, necesidades y potencialidades de cada territorio.

- el enfoque ascendente: se refiere a la implicación de los distintos agentes locales en la construcción de su propio proceso de desarrollo (enfoque bottom-up).

- los grupos de desarrollo rural: se destaca aquí el protagonismo de estos grupos, cuya composición, mecanismos de toma de decisiones, legitimidad, relaciones con otros agentes de desarrollo, implicación en el territorio..., son elementos que marcan la singularidad de estos programas respecto de otros.

- el carácter innovador de las acciones: se refiere a cuestiones tales como la promoción de nuevas formas de aprovechamiento de los recursos locales, el desarrollo de actuaciones que aporten soluciones a las deficiencias de la comarca, la definición e incorporación de tecnologías innovadoras y de todos aquellos elementos que supongan novedad respecto a otro tipo de programas.

- el enfoque integrado y multisectorial: se trata de la vinculación entre las diferentes actuaciones que se lleven a cabo en el territorio, de manera que se obtengan beneficios añadidos, tales como una mayor eficacia en las actuaciones o una mejor coordinación y cooperación entre agentes.

- la interconexión en red (regional, nacional e internacional): se busca con ello impulsar las relaciones de cooperación e intercambio entre los diferentes grupos de desarrollo a cualquier escala y por diferentes medios.

En Andalucía, ambos programas se aplican en 49 ámbitos territoriales (de alrededor de 100.000 habitantes cada uno de ellos), que cubren casi 700 municipios (el $89 \%$ del territorio de la Comunidad Autónoma andaluza) y agrupan a casi la mitad de la población, excluyendo sólo las ocho capitales de provincia, 
a los grandes núcleos urbanos de Jerez y Algeciras y a las zonas de litoral (ver Figura $\mathrm{n}^{\circ} 2$ ).

En cada ámbito territorial se aplica un programa específico de desarrollo cuya gestión corresponde a los grupos de desarrollo rural (en adelante, se mencionarán con las siglas GDR), que adoptan fórmulas jurídicas diversas (sociedades anónimas, consorcios y asociaciones, predominando estas últimas) ${ }^{8}$. Estos programas orientan el contenido de los proyectos de inversión en el territorio correspondiente, siendo aprobados por el GDR de acuerdo con criterios propios y con los más generales establecidos en las normativas reguladoras; una vez aprobados, los proyectos reciben una financiación, que procede de los fondos europeos destinados a cada programa ${ }^{9}$.

Figura $\mathrm{n}^{\circ} 2$

Grupos Leader II y Proder en Andalucía

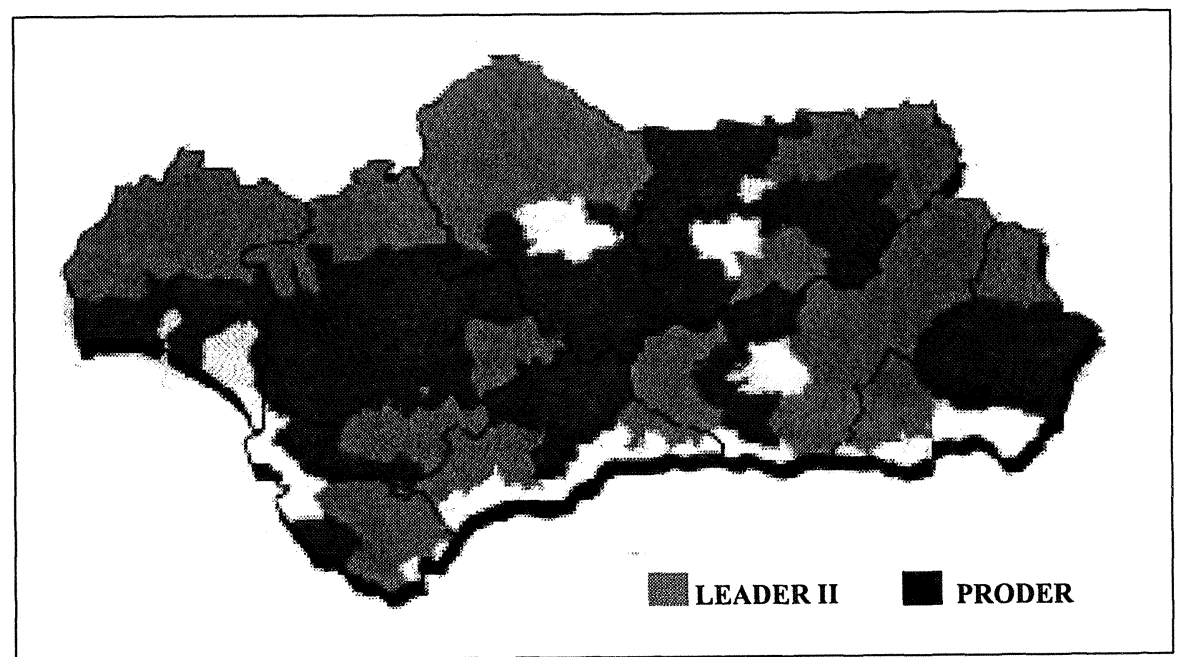

${ }^{8}$ Estos grupos se han venido denominando "grupos de acción local" en la iniciativa Leader (22 en Andalucía) y "grupos de desarrollo rural" en el programa Proder (27 grupos). Actualmente todos los grupos han debido adoptar la forma jurídica de asociación.

${ }^{9}$ La mayor parte de los Grupos se han establecido en áreas con identidades culturales fuertemente compartidas o bien en zonas donde el sentido de identidad no es tan fuerte, pero en las que hay rasgos territoriales comunes. Como resultado de la iniciativa Leader han surgido 131 nuevas organizaciones (de ellas 66 de carácter económico y 65 de carácter cultural: mujeres, jóvenes o ecologistas), produciendo, además, un incremento del número de los asociados preexistentes. Desde el punto de vista de las inversiones, 32.225 millones de pesetas (193,68 mill. Euros) se han invertido en proyectos aprobados dentro de la iniciativa Leader II y 25.996 millones de pesetas (156,24 mill. Euros), en el programa Proder. El origen de los fondos de financiación es diverso: la iniciativa privada representa el $40 \%$ de total de las inversiones en el Leader II y casi el $30 \%$ en el Proder, viniendo el resto de fondos de la UE, de acuerdo con las reglas de cofinanciación de las zonas Objetivo $\mathrm{n}^{\circ} 1$ donde se encuentra Andalucía. 


\section{RIS}

Por imperativo de la Comisión Europea, los programas Leader y Proder deben ser evaluados por agencias externas. Ha sido en la evaluación de la iniciativa Leader II y del programa Proder donde el IESA-CSIC participó analizando la opinión de la población andaluza, y ha sido en el marco de dicha evaluación, y como parte de ella, donde se han explorado algunas dimensiones del concepto de capital social. Este concepto destaca, tal como se ha señalado, la importancia, para el éxito de los programas de desarrollo, de recursos como la confianza entre vecinos, los flujos de información, las normas de reciprocidad existentes en una comunidad, la cooperación entre instituciones o la eficiencia de éstas en el ejercicio de sus funciones. De forma muy general, el concepto de capital social puede aproximarse a la idea de "competitividad social", que es definida por el Observatorio Europeo Leader como "la capacidad de los distintos agentes e instituciones para actuar de forma conjunta y eficaz en un territorio". Por tanto, es un concepto que se refiere a "la organización de los agentes locales y a las relaciones entre las personas (a nivel individual), entre agentes e instituciones y entre las propias instituciones"10. La operacionalización de dicho concepto de capital social se ha hecho definiendo distintos indicadores que hacen referencia a la confianza existente entre los vecinos de las diversas comarcas, al grado de participación de la población, a los lazos de comunicación entre asociaciones, a la confianza que tienen en las instituciones que les son más próximas y a la eficiencia que perciben en las actividades que éstas desarrollan. Estos indicadores se han medido mediante una encuesta a la población, cuyas bases metodológicas se explican en el siguiente apartado.

\section{Metodología}

Si bien se ha recurrido de forma complementaria a la información proporcionada por fuentes secundarias - fundamentalmente, para tener una visión de conjunto de cada una de las comarcas objeto de estudio-, el análisis de la opinión pública respecto a la iniciativa Leader II y al programa Proder se ha realizado a partir de la información facilitada directamente por la propia población. La recogida de dicha información se ha hecho mediante un programa de entrevistas grupales, a cuyos miembros se les distribuía el cuestionario para medir el capital social.

Para el conjunto de las 49 comarcas Leader II y Proder se ha entrevistado a 356 personas. De ellas, el $28 \%$ eran beneficiarias de alguno de los dos programas de desarrollo, el $13 \%$ eran empresarios no beneficiarios de dichos programas,

\footnotetext{
${ }^{10}$ Observatorio europeo LEADER (2000), La competitividad social. Construir una estrategia de desarrollo territorial con base en la experiencia de LEADER, Fascículo 2, Comisión Europea, Bruselas.
} 
el $14 \%$ pertenecía a asociaciones de carácter económico, el $21 \%$ procedía de asociaciones de ámbito sociocultural, el $11 \%$ eran representantes de los medios de comunicación, y el $13 \%$ provenía del sector educativo. En cuanto a las características de los entrevistados, aproximadamente el $62 \%$ eran hombres y el $38 \%$ mujeres, distribuidos en los siguientes grupos de edad: el 63\%, en el grupo de 30 y 45 años, el 16\%, menores de 29 años, y el $21 \%$ restante, en edades superiores a los 46 años. Siguiendo la Clasificación Nacional de Ocupaciones (CNO), el 45\% de los participantes en las entrevistas correspondía a la categoría de "dirección de empresas y de las administraciones públicas" (empresarios, dueños de comercio, dueños de bares...), siendo el segundo grupo en importancia el de los "técnicos y profesionales científicos e intelectuales" (médicos, maestros y profesores en general, periodistas...), con un $29 \%$, mientras que el tercero correspondía a los "artesanos y trabajadores cualificados de las industrias manufactureras, construcción y minería", con un $6 \%$. El restante $20 \%$ se repartía en otras categorías.

Para abordar la cuestión del capital social, y siguiendo el modelo reformulado de Woolcock, hemos tenido en cuenta cuatro dimensiones del mismo, a saber: a) la integración intracomunitaria, que se refiere a los lazos sociales dentro de la comunidad y a las relaciones que mantienen los individuos con miembros de su propia comunidad, pero distintos a los que componen sus grupos primarios; b) la conexión intercomunitaria, que hace referencia a la participación de los individuos en redes extracomunitarias y a su confianza en las instituciones que representan el interés general; c) la sinergia entre instituciones, que incluye la cooperación entre instituciones tanto públicas como privadas; y d) la eficiencia organizacional, que engloba lo relativo a la capacidad, competencia y credibilidad de las instituciones, y a su eficiencia en el ejercicio de las funciones que les son características ${ }^{11}$. La medición de esas cuatro dimensiones del capital social se ha hecho a través de un conjunto de indicadores que, en forma de 8 preguntas, constituyó la base del cuestionario distribuido entre las personas que asistieron a las entrevistas de grupo antes comentadas (ver anejo I).

Obviamente, de la información recogida de las 356 personas entrevistadas no pueden extraerse conclusiones estadísticamente representativas, pero sus respuestas tienen un valor cualitativamente interesante para el análisis, valor que se apoya en una metodología bastante utilizada en los estudios exploratorios y que utiliza lo que se denomina "muestra estratégica positiva"'2. Esta clase de muestras está

\footnotetext{
${ }^{11}$ Esta reformulación está basada en el trabajo ya citado de E. Moyano (2001), op. cit. pp.49.

${ }^{12}$ Una metodología similar a ésta fue utilizada por V. Pérez Díaz en su conocido estudio sobre las actitudes y estrategias de los agricultores castellanos ante la modernización de la agricultura (Pérez Díaz, 1983). En ese trabajo, Pérez Díaz señalaba que había "elegido una muestra relativamente favorable para confirmar la hipótesis de una transformación de los agricultores en empresarios agrarios. Si esto no se confirma en este caso, a fortiori será aún más improbable para el conjunto de los agricultores" (Pérez Díaz, 1983: 268). Era una "muestra estratégica positiva" sin pretensiones de representatividad estadística.
} 
formada por personas seleccionadas no aleatoriamente, sino mediante criterios definidos por el equipo investigador en función de la competencia de dichas personas para opinar sobre determinados temas y a su proclividad a hacerlo en un sentido positivo. El análisis parte de la idea de que las opiniones emitidas por la población incluida en la denominada "muestra estratégica" no son representativas de la población general, sino de una élite, y es precisamente ese carácter de élite el que permite deducir que si este grupo no percibe tan positivamente como pudiera esperarse determinados aspectos del tema objeto de estudio, es improbable que lo haga la población general.

El conjunto de las 356 personas que ha respondido al cuestionario sobre capital social constituye una "muestra estratégica positiva", por cuanto que, al haber estado formada por personas directamente comprometidas e implicadas en las dinámicas del desarrollo - bien por ser beneficiarias de proyectos, bien por pertenecer al mundo asociativo local, o bien por proceder del mundo educativo-, debiera esperarse que perciban el nivel del capital social en el mundo rural andaluz de un modo más positivo que el conjunto de la población general. Es decir, si la población incluida en la muestra estratégica no percibe que haya, por ejemplo, un buen nivel de integración o de sinergia en la sociedad rural andaluza, puede esperarse que la población general tampoco lo percibirá. Caso diferente sería si la población entrevistada percibiera la existencia de altos niveles de capital social en el mundo rural de Andalucía, pues entonces no podría extenderse - precisamente, por su carácter de muestra estratégica positiva - tal percepción al conjunto de la población. En definitiva, sus respuestas han sido valoradas como lo que son, es decir, como las de un grupo de referencia cuyas opiniones reflejan la percepción de una élite y no la de la población general que reside en los territorios rurales de Andalucía. Con esta premisa se procederá a esta parte del análisis.

\section{Capital social y dinámicas de desarrollo en las zonas rurales andaluzas}

De acuerdo con el planteamiento metodológico señalado al comienzo de este artículo, se han analizado cuatro dimensiones del capital, a saber: integración intracomunitaria, conexión intercomunitaria, sinergia entre instituciones y eficiencia organizacional (ver tabla 1).

Antes de proceder a presentar los resultados del análisis conviene detenerse en un aspecto importante. Nos referimos al problema que se plantea al utilizar escalas ordinales de 1 a 5 -en las que 1 indica el valor mínimo y 5 el valor máximo - para medir aspectos como la confianza, el grado de participación y cooperación o la eficiencia. La tendencia de la población es a evitar las posiciones extremas, de ahí que los valores medios se sitúen en torno al 3 debido a que la mayor parte de los entrevistados se sitúan en las posiciones 2 (poca confianza, poca cooperación o poca eficiencia), 3 (alguna confianza, alguna cooperación o alguna eficiencia) y 4 (bastante confianza, bastante cooperación o bastante efi- 
ciencia). La interpretación de los resultados se ha realizado agrupando, de un lado, los porcentajes correspondientes a las posiciones 1 (ninguna) y 2 (poca) - como indicativos de un nivel bajo de confianza, cooperación o eficiencia- $y$, de otro, los correspondientes a las posiciones 4 (bastante) y 5 (mucha) - como indicativos de un nivel alto de confianza, cooperación o eficiencia-. El problema se plantea al interpretar las opiniones de los individuos que optan por la posición 3 (alguna), y que suelen ser precisamente los que arrojan los porcentajes más elevados. Un análisis exigente que tuviera en cuenta el carácter de "muestra estratégica positiva" de la población entrevistada, tendería a considerar esos porcentajes como representativos de un bajo nivel de capital social; en efecto, una interpretación de este tipo no consideraría como indicativo de una buena valoración el hecho de que un grupo de élite elija la opción 3 (alguna), en vez de las opciones 4 (bastante) y 5 (mucha). Un análisis menos exigente podría valorar en términos positivos que un sector de la población entrevistada se haya situado en esta posición intermedia en vez de en las posiciones mínimas 1 (ninguna) y 2 (poca). En nuestro estudio nos hemos inclinado por interpretar los resultados del siguiente modo: hay un nivel alto de capital social cuando la suma de los porcentajes situados en las posiciones 4 y 5 supera el $75 \%$ del total de la población entrevistada; hay un nivel medioalto cuando dicho porcentaje supera el $50 \%$; hay un nivel medio-bajo cuando no alcanza el $50 \%$, pero supera la suma de los porcentajes de las posiciones 1 y 2 ; y hay un nivel bajo si no alcanza el $25 \%$ ni supera al de estas posiciones mínimas extremas.

Con estas premisas, pueden hacerse los siguientes comentarios. En primer lugar, y en lo que respecta a la dimensión de integración intracomunitaria - entendida como la confianza que tienen los individuos en sus vecinos a la hora de emprender proyectos de carácter colectivo-, la población entrevistada muestra un nivel que podríamos considerar medio-bajo, manifestado a través de

Tabla 1.

Dimensiones e indicadores del concepto de capital social

\begin{tabular}{ll}
\hline DIMENSIONES & \multicolumn{1}{c}{ INDICADORES } \\
\hline Integración intracomunitaria & $\begin{array}{l}\text { Grado de confianza entre vecinos } \\
\text { Grado de participación en proyectos colectivos } \\
\text { Grado de identidad comarcal } \\
\text { Grado de confianza que inspiran las instituciones }\end{array}$ \\
Conexión intercomunitaria & $\begin{array}{l}\text { Grado de cooperación entre instituciones } \\
\text { Grado de cooperación entre municipios } \\
\text { Efinergia institucional }\end{array}$ \\
Eficiencia organizacional & Eficiencia de las instituciones públicas \\
\hline
\end{tabular}


RIS

REVISTA INTERNACIONAL DE SOCIOLOGIA

No 33, Septiembre-Diciembre, 2002

FERNANDO E. GARRIDO FERNÁNDEZ y EDUARDO MOYANO ESTRADA

dos indicadores: el grado de confianza entre vecinos que los entrevistados perciben en sus municipios, y el grado de participación en actividades de tipo colectivo que éstos les atribuyen. Así, respecto al primer indicador, el 33,5\% de entrevistados que dicen que en su municipio existe bastante o mucha confianza entre vecinos, no alcanza el $50 \%$, pero supera el $19,1 \%$ de los que dicen que hay ninguna o poca confianza. Utilizando los valores de la escala de 1 a 5 , podemos señalar que, en lo que se refiere a este primer indicador, la población entrevistada se sitúa en el 3,22, es decir, en una posición intermedia. Respecto al segundo indicador, el nivel es también medio-bajo, ya que el porcentaje de los que consideran que los vecinos de su municipio participan mucho o bastante en actividades de tipo colectivo no alcanza la mitad de la población entrevistada (sólo llega al 38,2\%), pero supera al de los que opinan que participan poco o nada $(25,5 \%)$. Tomando como referencia la escala de 1 a 5 utilizada en el cuestionario, la población se sitúa en una posición muy parecida a la del primer indicador (un 3,20). En resumen, si esa percepción de lo que ocurre en sus municipios la proyectamos sobre los propios ciudadanos, no parece que los andaluces que residen en las zonas rurales tengan gran confianza en sus vecinos. Este dato es aún más revelador del bajo nivel de integración existente si tenemos en cuenta que la población entrevistada está formada por un grupo relativamente selecto de personas que han mostrado una disposición positiva a colaborar en proyectos colectivos - lo que hemos denominado una "muestra estratégica"-. Si este grupo no percibe un nivel relativamente alto de integración en sus municipios, es improbable que la población general lo perciba, de donde puede deducirse que, en el conjunto de la población, la percepción de la confianza entre vecinos será aún más baja que en la muestra estratégica.

En segundo lugar, la dimensión de conexión intercomunitaria —entendida como las relaciones de confianza que mantiene la población con individuos, grupos e instituciones que no pertenecen a su comunidad primaria (es decir, distintas de sus familias, amigos o vecinos) y que trascienden el ámbito restringido de la localidad de residencia - se ha medido en nuestro estudio a través de dos indicadores: el grado de identidad comarcal percibida por la población (la idea de pertenecer a una comarca común, más allá del pueblo de residencia), y el grado de confianza que inspiran determinadas instituciones (como el propio Grupo de Desarrollo Rural, las entidades financieras, el Instituto de Fomento de Andalucía (IFA), los ayuntamientos, las diputaciones provinciales o la Junta de Andalucía). Respecto al primer indicador, puede decirse que la población percibirá que existe identidad comarcal si considera que hay cooperación entre las poblaciones de los distintos municipios de la comarca. De acuerdo con este planteamiento, se observa que el nivel de identidad comarcal percibido por la población en los municipios de su correspondiente comarca, es medio-bajo, ya que menos de la mitad (un 39,0\%) considera que existe mucha o bastante identidad comarcal, pero supera al $32,5 \%$ que dice que existe poca o ninguna. En valores medios de la escala, este indicador 
$\begin{array}{r}\text { R IS } \\ \text { CAPITAL SOCIAL Y DESARROLLO EN ZONAS RURALES } \\ \hline\end{array}$

arroja un valor de 3,11 (en la escala de 1 a 5). Respecto al segundo indicador, y considerando el conjunto de las instituciones presentadas a los entrevistados, puede decirse que la población rural de Andalucía muestra también un nivel medio-bajo (un 3,25 en una escala de 1 a 5): menos de la mitad (37,5\%) de los entrevistados ha indicado tener bastante o mucha confianza en las instituciones con las que se relaciona, superando al $17,1 \%$ que ha expresado abiertamente que tiene poca o ninguna confianza. Si tenemos en cuenta que no todas las instituciones están teniendo la misma importancia como factores impulsores del desarrollo, y que no todas tienen una presencia similar en las dinámicas que se desarrollan en las distintas comarcas, podemos centrar el análisis en aquéllas cuya importancia, a la luz de la información disponible, ha sido mayor durante el periodo de vigencia de la iniciativa Leader II y del programa Proder, como por ejemplo los grupos de desarrollo rural (GDR), los ayuntamientos y la Junta de Andalucía, excluyendo al IFA (sólo presente en algunas comarcas), a las mancomunidades de municipios (cuya existencia no está generalizada en el conjunto del territorio andaluz), a las diputaciones provinciales (cuyo protagonismo ha sido escaso) y a las entidades financieras (cuya implicación en la financiación de los proyectos ha sido baja). Centrando el análisis en la confianza que inspiran esas tres instituciones, se observa que el nivel de esta dimensión del capital social aumenta respecto a la media general: pasa del 3,18 al 3,40, con valores máximos en los GDR (con un 3,87), y en los ayuntamientos (con un 3,49). Es preocupante para la sostenibilidad de las dinámicas de desarrollo, e indicativo del reducido nivel de capital social todavía existente en la sociedad rural andaluza, que instituciones cuya función para la promoción del desarrollo debería ser importante - como las mancomunidades de municipios, las diputaciones o las entidades financieras-inspiren un bajo nivel de confianza en la población - todas ellas por debajo de la media general: 3,06; 3,03 y 2,59 , respectivamente.

Tabla 2.

Percepción del capital social de comarcas Leader-Proder de Andalucia, según dimensión

\begin{tabular}{lcc}
\hline DIMENSIONES & INDICADORES & NIVEL \\
\hline $\begin{array}{l}\text { Integración } \\
\text { intracomunitaria }\end{array}$ & Confianza entre vecinos & Medio-Bajo \\
$\begin{array}{l}\text { Conexión } \\
\text { intercomunitaria }\end{array}$ & Identidad comarcal & Medio-Bajo \\
Sinergia & Confianza instituciones & Medio-Bajo \\
institucional & Cooperación asociaciones & Medio-Bajo \\
Eficiencia & Cooperación instituciones & Bajo \\
organizacional & & Bajo \\
\hline
\end{tabular}


RIS

REVISTA INTERNACIONAL DE SOCIOLOCIA

$N^{\bullet} 33$, Septiembre-Diciembre, 2002

FERNANDO E. GARRIDO FERNÁNDEZ y EDUARDO MOYANO ESTRADA

En tercer lugar, la dimensión de sinergia institucional —entendida como el nivel de cooperación entre instituciones percibido por la población - se ha medido a través de dos indicadores: el primero, se refiere al grado de cooperación entre instituciones del ámbito asociativo, y el segundo, hace referencia al grado de cooperación entre municipios (personificados en la figura del Ayuntamiento). De acuerdo con la información obtenida en las entrevistas, ambos indicadores presentan valores bajos ( 3,01 y 3,02 , respectivamente, en una escala de 1 a 5$)$, de forma que, conjuntamente, alcanzan sólo el 3,02. Analizando los datos en terminos porcentuales, puede decirse que, en lo que se refiere al primer indicador, se percibe un nivel medio-bajo (muy cerca del nivel bajo) de comunicación entre asociaciones, tal como lo corrobora el hecho de que sea mayor el porcentaje de los que creen que hay poca o ninguna comunicación interasociativa (un 34,0\%), que el de los que consideran que hay bastante o mucha comunicación (un $28,9 \%$, que apenas supera el límite del 25\%). Respecto al segundo indicador, la percepción de la cooperación intermunicipal es aún más baja que en el caso de las asociaciones, superando apenas la cuarta parte de los entrevistados (un 28,6\%) el porcentaje de los que consideran que hay mucha o bastante cooperación entre los diferentes municipios, y siendo superados por el de los que creen que hay poca o ninguna cooperación $(30,6 \%)$.

En cuarto y último lugar, la dimensión de eficiencia organizacional —entendida como el grado de eficiencia de las instituciones públicas y privadas en el ejercicio de sus funciones y en la prestación de servicios, tal como son percibidas por la población - se ha analizado a través del correspondiente indicador. Al igual que en la dimensión de integración, este indicador se ha referido tanto a la eficiencia de las instituciones en general, como a cada institución en particular. En lo que se refiere a las seis instituciones seleccionadas, la percepción de eficiencia desciende ligeramente respecto a la de confianza (un 3,22 en una escala de 1 a 5), situándose en un nivel medio-bajo, ya que el porcentaje de la población entrevistada que considera que las instituciones son bastante o muy eficientes en sus actividades no alcanza el $50 \%$ (se sitúa en un $31,4 \%$ ), pero supera al grupo de los que consideran que son poco o nada eficientes (14,2\%). Si seleccionamos las tres instituciones con mayor presencia en las dinámicas del desarrollo local, el valor de la percepción se eleva al 3,29 (con un 3,78 para los grupos de desarrollo rural, un 3,21 para los ayuntamientos y un 3,10 para la Junta de Andalucía). En porcentajes, son los grupos de desarrollo rural los percibidos como más eficientes por un mayor número de entrevistados (un $54,5 \%$ de la población cree que son bastante o muy eficientes lo que los sitúa en un nivel alto), seguidos de los ayuntamientos (con un $39,1 \%$, en un nivel medio-bajo) y de la Junta de Andalucía (con un 27,5\%, en un nivel bajo). Resulta preocupante que instituciones llamadas a cumplir una función importante para garantizar la continuidad de las dinámicas de desarrollo impulsadas desde las iniciativas Leader y Proder (como las mancomunidades o las diputaciones) sean percibidas como poco eficientes por la población entrevis- 
tada, no alcanzando ninguna de ellas el aprobado (2,99 y 2,93, respectivamente). Los porcentajes corroboran esta apreciación, ya que son las diputaciones las que reúnen el menor número de entrevistados que consideran que son bastante o muy eficientes (sólo un $22,5 \%$ así lo cree, frente al $27,3 \%$ que cree que son poco o nada eficientes).

En definitiva, a la luz de este análisis experimental del capital social puede decirse que la población entrevistada no percibe unos niveles altos en ninguna de sus cuatro dimensiones, sino niveles medios o medios-bajos. Ello permite deducir que, dado el carácter estratégico de la muestra, es improbable que la población andaluza que reside en las zonas rurales afectadas por los programas Leader y Proder tenga una percepción mejor del capital social que la expresada por los entrevistados. Si le damos importancia a este concepto como indicativo del estado de vertebración y aprovechamiento de los recursos no económicos y valoramos su influencia en el éxito de los procesos de desarrollo rural, hemos de reconocer que, independientemente de lo que nos digan otros indicadores objetivos (número de proyectos aprobados, generación de empleo, recursos invertidos, ...), los valores obtenidos en nuestro estudio son preocupantes, ya que muestran el bajo nivel de vertebración social, de cooperación intermunicipal y de eficiencia institucional de los territorios rurales de Andalucía tal como es percibido por la población.

No obstante, dado el escaso tiempo transcurrido desde la aplicación de los programas de desarrollo rural (diez años de la iniciativa Leader y cuatro del programa Proder) y debido a que se trata de programas-piloto para dinamizar las zonas rurales, los resultados de nuestro análisis deberían interpretarse a la luz del contexto general, de tal modo que un nivel medio-bajo de capital social puede ser interpretado en términos positivos si está significando un avance respecto a situaciones de partida en las que algunas de sus dimensiones (como las referidas a la identidad comarcal o la cooperación intermunicipal) alcanzaban niveles muy bajos. Puede decirse, en resumen, que el capital social de las zonas rurales está siendo inducido por las políticas de desarrollo, unas políticas cuya eficacia futura dependerá de su contribución a aumentar aquellas dimensiones que garanticen la sostenibilidad del proceso de desarrollo, resolviendo así no sólo los dilemas estáticos de la cooperación, sino también sus dilemas dinámicos.

\section{CONCLUSIONES}

En este artículo se ha aplicado el enfoque del capital social al estudio de las dinámicas de desarrollo en zonas rurales de Andalucía. El interés del trabajo radica en hacer operativo un concepto polisémico como el de capital social y mostrar su utilidad para el análsisis de experiencias concretas donde el factor clave es la capacidad de los individuos para cooperar en pos de proyectos de carácter colectivo en beneficio de su comunidad. Utilizando el modelo ampliado de M. Woolcock y 
reformando algunas de sus dimensiones, se han elaborado varios indicadores para medir el nivel de capital social en las zonas rurales de Andalucía, aprovechándose la oportunidad brindada por la participación del IESA-CSIC en la evaluación de los programas Leader II y Proder de esa Comunidad Autónoma. La sintonía de los objetivos de dichos programas con las dimensiones del capital social convierte a este enfoque teórico en una perspectiva idónea para el análisis de las dinámicas de desarrollo generadas en esos territorios.

Dadas las características de la evaluación (diseñada para medir aspectos de los programas Leader y Proder no relacionados directamente con las dimensiones del capital social), los resultados presentados en este trabajo son los de un análisis experimental sobre las posibilidades de operativizar el concepto de capital social. La selección de una muestra estratégica convierten tales resultados en indicativos de lo que piensa una élite de la población rural de Andalucía, pero no el conjunto de esa población. No obstante, la información proporcionada es de gran utilidad para comprender las dinámicas de desarrollo generadas por los programas Leader y Proder. Del análisis realizado pueden extraerse lecciones sobre la idoneidad de los indicadores utilizados, para, a partir de ellas, ver la posibilidad de extender su aplicación a muestras representativas de la población.

\section{BIBLIOGRAFÍA}

BANDFIELD, E. (1958), The Moral Basis of a Backward Society, Nueva York, Free Press.

BOIX, C. y D. N. POSTER (1996 y 2000), "Making Social Capital Work. A review of Robert Putnam's Making Democracy Work: Civic Traditions in Modern Italy", Working Paper Series 96-4 (Cambridge: Harvard University Centre for International Affairs). Hay una versión española de este artículo en Revista Española de Ciencia Politica, vol. 1, n 2, pp. 159-185.

BOURDIEU, P. (1986), “The forms of capital”, in John Richardson (ed.), Handbook of Theory and Research for the Sociology of Education, Westport, Greenwood Press.

BOURDIEU, P. y J.C. PASSERON (1990), Reproduction in Education, Society and Culture, Londres, Sage, $1^{\mathrm{a}}$ versión en francés en 1970.

BURT, R. (1992), Structural Holes, Cambridge, Harvard University Press.

COLEMAN, J. (1988), "Social capital in the creation of human capital", American Journal of Sociology, 94, pp. S95-S120.

(1990), Foundations of Social Theory, Cambridge, Harvard University Press.

DE FRANCISCO, A. y F. HERREROS (2001), "Capital social”, Zona Abierta (número monográfico), Madrid, Fundación Pablo Iglesias.

ETZIONI, A. (1993), The Spirit of Community, Nueva York, Crown Publishers. 
EVANS, P. (1992), "The State as problem and solution: embedded autonomy, and structural change", in S. Haggard and R. Kauffman (eds.), The Politics of Economic Adjustment, Princeton, Princeton University Press.

(1995), Embeddedness Autonomy, Princeton, Princeton University Press.

(1996), "Government Action, Social Capital and Development: Reviewing the Evidence on Synergy”, World Development, n 24/6, pp.1122.

FEDDDERKE, J. et al. (1999), "Economic Growth and Social Capital. A critical reflection", Theory and Society, vol. 28/5, pp. 709-745.

FOSTER, G. (1965), "Peasant society and the image of limited good", American Anthropologist, $\mathrm{n}^{\circ} 67$, pp. 293-315.

FUKUYAMA, F. (1995), Trust: the Social Virtues and the Creation of Prosperity, Nueva York, The Free Press.

GREIF, A. (1993), "Contract enforceability and economic institutions in early trade", American Economic Review, $\mathrm{n}^{\circ} 83$, pp. 525-549.

(1994), "Cultural beliefs and the organization of society: a historical and theoretical reflection on collectivist and individualist societies", Journal of Political Economy, $\mathrm{n}^{\circ} 102, \mathrm{pp} .912$ 950.

GRANOVETTER, M. (1973), "The strength of weak ties", American Journal of Sociology, $\mathrm{n}^{\circ}$ 78, pp. $1360-1380$.

(1985), "Economic action and social structure: the problem of embeddedness", American Journal of Sociology, n 91, 493.

HIRSCHMANN, A. (1958), The Strategy of Economic Development, New Haven, Yale University Press.

HERREROS, F. (2001), "Social capital, associations and civic republicanism”, en M. Saward (ed.), Democratic Innovation, Deliberation, Representation and Association, Londres, Routledge.

(2002a), Capital social y confianza, Tesis doctoral, Universidad Complutense de Madrid, Madrid.

(2002b), ¿Son las relaciones sociales una fuente de recursos?. Una definición de capital social”, Papers, $\mathrm{n}^{\circ}$ 67, pp. 129-148.

JACOBS, J. (1961), The Life and Death of the Great American Cities, Nueva York, Random House.

LOURY, G. (1977a), "The economic of discrimination: getting to the core of the problem", Harvard Journal of African American Public Policy, $\mathrm{n}^{\circ} 100$.

(1977b), “A dynamic theory of racial income differences", en P.A. Wallace y A. le Mund (eds.), Women, Minorities and Employment Discrimination, Lexington, Lexington Books. 
RIS

REVISTA INTERNACIONAL DE SOCIOLOCIA

MIGDAL, J. (1988), Strong Societies and Weak States: State-Society Relations and State Capabilities in the Third World, Princeton, Princeton University Press.

MONTERO, J. R. y M. TORCAL (2000), "La formación y consecuencias del capital social”, Revista Española de Ciencia Politica, vol. 1, n² 2, pp. 79-121.

MOYANO, E. (2001), "El enfoque del capital social y su utilidad para el análisis de las dinámicas del desarrollo", Revista de Fomento Social, n 56, pp. 35-63.

OLSON, M. (1965), The Logic of Collective Action, Cambridge, University Press.

(1982), The Rise and Decline of Nations, New Haven, Yale University Press.

PEREZ DIAZ, V. (1983), "Los nuevos agricultores", Papeles de Economia Española, n 16, pp. 240-268.

POLANYI, K. (1957), The Great Transformation, Boston, Beacon Press.

PORTES, A. (1998), "Social capital: Its Origins and Aplication in Modern Sociology", American Review of Sociology, $\mathrm{n}^{\circ}$ 24, pp. 1-24.

PORTES, A. (ed.) (1995), The Economic Sociology of Immigration: Essays on Networks, Ethnicity and Entrepreneurship, New York, Russel Sage Foundation.

PORTES, A. y P. LANDOLT (1996), "The downside of social capital", The American Prospect, $\mathrm{n}^{\circ}$ 26, pp. 18-21.

PORTES, A. y J. SENSENBRENNER (1993), "Embeddedness and immigration: notes on the social determinants of economic action", American Journal of Sociology, n 98/6, pp. 1320-1350.

PUTNAM, R. (1993), Making Democracy Work, Princeton, Princeton Univ. Press.

(1995), "Bowling alone. American's declining social capital", Journal of Democracy, $n^{\circ} 6 / 1$, pp. 65-78.

RUESCHEMEYER, D. y P. EVANS (1985), "The state and economic transformation", en P. Evans, D. Rueschemeyer y T. Skocpol (eds.), Bringing the State Back in, Nueva York, Cambridge University Press, $\mathrm{n}^{\circ} 59$.

SCHAMBRA, W. (1994), "By the people: the old values of the new citizenship", Policy Review, summer, $\mathrm{n}^{\circ}$ 32-38.

SCHULTZ, T. W. (1963), "Investment in human capital”, American Economic Review, no 51, pp. $1-16$.

SEN, A. (1981), Poverty and Famines: An Essay on Entitlement and Deprivation, NuevaYork, Oxford University Press.

SKOCPOL, T. (1995), Social Policy in the United States: Future Possibilities in Historical Perspectives, Princeton, Princeton University Press. 
SWEDBERG, R. (1991), "Major traditions of economic sociology", Annual Review of Sociology, $\mathrm{n}^{\circ} 17,251-276$.

SWEDBERG, R. y N. SMELSER (eds.) (1994), Handbook of Economic Sociology, Princeton, Princeton University Press.

WOOLCOCK, M. (1998), "Social capital and economic development: toward a theoretical synthesis and policy framework", Theory and Society, $\mathrm{n}^{\circ} 27, \mathrm{pp} .151-208$.

ZARTMAN, I. (ed.) (1995), Collapsed States: The Disintegration and Restoration of Legitimate Authority, Boulder, Lynne Rienner Publishers. 
RIS

REVISTA INTERNACIONAL DE SOCIOLOGIA

№33, Septiembre-Diciembre, 2002

FERNANDO E. GARRIDO FERNÁNDEZ y EDUARDO MOYANO ESTRADA

\section{ANEJO I \\ Cuestionario sobre capital social}

P1. En general, ¿podría decirnos en qué medida cree Ud. que existe confianza entre los vecinos de su municipio?

$\begin{array}{llllll}\frac{\text { Ninguna }}{\text { confianza }} & & 3 & & \frac{\text { Mucha }}{\text { confianza }} & \frac{\text { NS/ }}{\text { NC }}\end{array}$

P2. En su opinión, los vecinos de su municipio, ¿en qué medida participan en las distintas actividades (culturales, deportivas, políticas...) que se organizan?

$\begin{array}{lllll}\frac{\text { Ninguna }}{\text { participación }} & & & \frac{\text { Mucha }}{\text { participación }} & \frac{\text { NS/ }}{\text { NC }}\end{array}$

P3. ¿Hasta qué punto cree Ud. que existe identidad comarcal (idea de pertenencia a una comarca común) entre los distintos municipios de su zona?

\begin{tabular}{|c|c|c|}
\hline Baja identidad & & dentidad \\
\hline comarcal & & \\
\hline 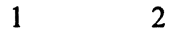 & 3 & \\
\hline
\end{tabular}

P4. Y sobre las asociaciones de todo tipo que están presentes en su municipio y que Ud. conozca, ¿en qué medida cree Ud. que hay comunicación entre ellas?

$\begin{array}{lllll}\frac{\text { Ninguna }}{\text { comunicación }} & 3 & 4 & \frac{\text { Mucha }}{\text { comunicación }} & \frac{\mathrm{NS} /}{5}\end{array}$

P5. ¿Hasta qué punto cree Ud. que las asociaciones que existen en su municipio constituyen un factor importante para el desarrollo de su comarca?

$\begin{array}{lllll}\frac{\text { Nada }}{\text { importantes }} & & & \frac{\text { Muy }}{\text { importantes }} & \frac{\text { NS/ }}{5}\end{array}$

P6. Si pensamos ahora en la comarca en la que Ud. reside, ¿podría valorar el grado de cooperación que existe entre los diferentes municipios de la comarca?

$\begin{array}{lllll}\frac{\text { Ninguna }}{1} & & \frac{\text { Mucha }}{\text { cooperación }} & \frac{\text { NS/ }}{5}\end{array}$

90 
P7. De las siguientes instituciones que le cito, ¿podría valorarme, en términos generales, qué grado de confianza le merecen?

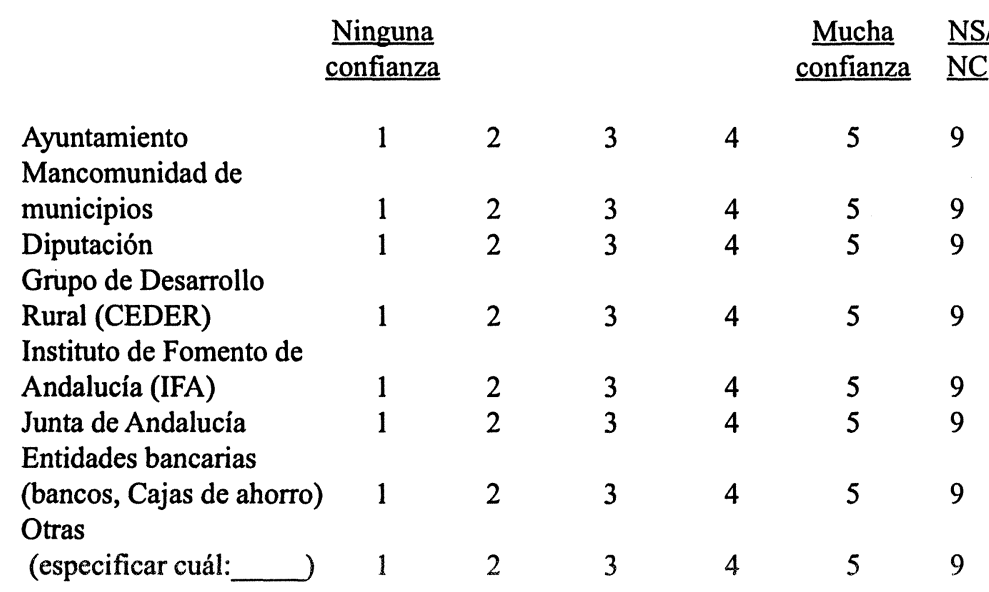

P8. En general, ¿considera Ud. a estas instituciones eficientes en sus actividades?

Nada

eficientes

\section{Ayuntamiento}

Mancomunidad de municipios

Diputación

Grupo de Desarrollo

Rural (CEDER)

Instituto de Fomento de

Andalucía (IFA)

Junta de Andalucía

Entidades bancarias

(bancos, Cajas de ahorro)

Otras

(especificar cuál:

$1-2=3-4=0$

$\begin{array}{llllll}1 & 2 & 3 & 4 & 5 & 9\end{array}$

$\begin{array}{llllll}1 & 2 & 3 & 4 & 5 & 9\end{array}$

$\begin{array}{lllllll}1 & 2 & 3 & 4 & 5 & 9\end{array}$

$\begin{array}{llllll}1 & 2 & 3 & 4 & 5 & 9\end{array}$

$\begin{array}{llllll}1 & 2 & 3 & 4 & 5 & 9\end{array}$

$\begin{array}{lllllll}1 & 2 & 3 & 4 & 5 & 9\end{array}$

$\begin{array}{lllllll}1 & 2 & 3 & 4 & 5 & 9\end{array}$


RIS

REVISTA INTERNACIONAL DE SOCIOLOGIA

\section{ANEJO 2}

Capital Social: Tablas de resultados

\section{INTEGRACIÓN INTRACOMUNITARIA}

En general, ¿podría decirnos en qué medida cree Ud. que existe relación de confianza entre los vecinos de su municipio?

Tabla P1

\begin{tabular}{llr}
\hline Confianza en vecinos & Ninguna confianza & $2,2 \%$ \\
& 2 & $16,9 \%$ \\
& 3 & $44,7 \%$ \\
TOTALES & 4 & $23,9 \%$ \\
& Mucha confianza & $9,6 \%$ \\
& NS/NC & $2,8 \%$ \\
& & 356 \\
& & $100 \%$ \\
& Media & 3,22 \\
& Desv. típica & 0,93 \\
\hline
\end{tabular}

¿Considera Ud. que los vecinos de su municipio participan en las distintas actividades (culturales, deportivas, políticas...) que se organizan?

Tabla P2

\begin{tabular}{llr}
\hline Participación & Ninguna participación & $0,8 \%$ \\
& 2 & $24,7 \%$ \\
& 3 & $34,3 \%$ \\
TOTALES & 4 & $30,1 \%$ \\
& Mucha participación & $8,1 \%$ \\
& NS/NC & $2,0 \%$ \\
& & 356 \\
& Media & $100 \%$ \\
& Desv. típica & 3,20 \\
& & 0,94 \\
\hline
\end{tabular}

92 


\section{CONEXIÓN INTERCOMUNITARIA}

¿Hasta qué punto cree Ud. que existe identidad comarcal (idea de pertenecer a una comarca común) entre los distintos municipios de su zona?

Tabla P3

\begin{tabular}{llr}
\hline Identidad comarcal & Ninguna identidad & $8,1 \%$ \\
& 2 & $24,4 \%$ \\
& 3 & $27,0 \%$ \\
TOTALES & 4 & $26,1 \%$ \\
& Mucha identidad & $12,9 \%$ \\
& NS/NC & $1,4 \%$ \\
& & 356 \\
& & $100 \%$ \\
& Media & 3,11 \\
& Desv. Típica & 1,17 \\
\hline
\end{tabular}

¿Podría valorarme, en términos generales, qué grado de confianza le merecen las instituciones...?

Tabla P7

\begin{tabular}{llr}
\hline Confianza en instituciones & Ninguna confianza & $0,8 \%$ \\
& 2 & $16,3 \%$ \\
& 3 & $45,4 \%$ \\
\multirow{3}{*}{ TOTALES } & 4 & $32,1 \%$ \\
& Mucha confianza & $5,4 \%$ \\
& & 356 \\
& & $100 \%$ \\
& Media & 3,25 \\
& Desv. típica & 0,82 \\
\hline
\end{tabular}


Tabla P7 bis:

Confianza en instituciones

\begin{tabular}{|c|c|c|c|c|c|c|c|}
\hline & Ayto. & $\begin{array}{l}\text { Manc. } \\
\text { Munic. }\end{array}$ & Diputación & GDR & IFA & $\begin{array}{c}\text { Junta } \\
\text { Andalucía }\end{array}$ & $\begin{array}{c}\text { Entidad } \\
\text { Bancaria }\end{array}$ \\
\hline $\begin{array}{l}\text { Ninguna } \\
\text { confianza }\end{array}$ & $7,0 \%$ & $5,6 \%$ & $7,9 \%$ & $2,0 \%$ & $7,0 \%$ & $5,6 \%$ & $18,0 \%$ \\
\hline 2 & $11,5 \%$ & $21,3 \%$ & $22,2 \%$ & $6,2 \%$ & $19,7 \%$ & $18,8 \%$ & $27,2 \%$ \\
\hline 3 & $27,8 \%$ & $23,6 \%$ & $29,5 \%$ & $21,3 \%$ & $30,1 \%$ & $33,7 \%$ & $28,9 \%$ \\
\hline 4 & $29,5 \%$ & $19,1 \%$ & $23,9 \%$ & $27,8 \%$ & $18,0 \%$ & $23,3 \%$ & $16,3 \%$ \\
\hline $\begin{array}{l}\text { Mucha } \\
\text { confianza }\end{array}$ & $21,9 \%$ & $9,0 \%$ & $8,4 \%$ & $28,1 \%$ & $9,8 \%$ & $9,6 \%$ & $3,9 \%$ \\
\hline $\mathrm{NS} / \mathrm{NC}$ & $2,2 \%$ & $21,3 \%$ & $8,1 \%$ & $14,6 \%$ & $15,4 \%$ & $9,0 \%$ & $5,6 \%$ \\
\hline \multirow[t]{2}{*}{ TOTALES } & 356 & 356 & 356 & 356 & 356 & 356 & 356 \\
\hline & $100 \%$ & $100 \%$ & $100 \%$ & $100 \%$ & $100 \%$ & $100 \%$ & $100 \%$ \\
\hline Media & 3,49 & 3,06 & 3,03 & 3,87 & 3,05 & 3,14 & 2,59 \\
\hline Desv. típica & 1,17 & 1,12 & 1,10 & 1,03 & 1,12 & 1,06 & 1,11 \\
\hline
\end{tabular}

\section{SINERGIA INSTITUCIONAL}

De las asociaciones de todo tipo que están presentes en su municipio y que Ud. conozca. ¿en qué medida cree Ud. que hay comunicación entre ellas?

\section{Tabla P4}

\begin{tabular}{llr}
\hline Comunicación entre asociaciones & Ninguna comunicación & $2,5 \%$ \\
& 2 & $31,5 \%$ \\
& 3 & $32,0 \%$ \\
TOTALES & 4 & $19,9 \%$ \\
& Mucha comunicación & $9,0 \%$ \\
& NS/NC & $5,1 \%$ \\
& & 356 \\
& & $100 \%$ \\
& Media & 3,01 \\
& Desv. típica & 1,01 \\
\hline
\end{tabular}


Si pensamos ahora en la comarca en la que Ud. reside, ¿podría valorar el grado de cooperación que existe entre los diferentes municipios de la comarca?

\begin{tabular}{llr}
\multicolumn{2}{c}{ Tabla P6 } \\
\hline Cooperación municipios & Ninguna cooperación & $4,5 \%$ \\
& 2 & $26,1 \%$ \\
& 3 & $35,7 \%$ \\
TOTALES & 4 & $19,9 \%$ \\
& Mucha cooperación & $8,7 \%$ \\
& NS/NC & $5,1 \%$ \\
& & 356 \\
& & $100 \%$ \\
& Media & 3,02 \\
& Desv. Típica & 1,02 \\
\hline
\end{tabular}

\section{EFICIENCIA ORGANIZACIONAL}

En general, ¿considera Ud. a las instituciones eficientes en sus actividades?

\begin{tabular}{llr}
\multicolumn{2}{c}{ Tabla P8 } \\
\hline Eficiencia instituciones & Ninguna eficiencia & $1,1 \%$ \\
& 2 & $12,9 \%$ \\
& 3 & $54,6 \%$ \\
TOTALES & 4 & $26,0 \%$ \\
& Mucha eficiencia & $5,4 \%$ \\
& & \\
& & 356 \\
& Media & $100 \%$ \\
& Desv. Típica & 3,22 \\
& & 0,78 \\
\hline
\end{tabular}




\section{RIS}

REVISTA INTERNACIONAL DE SOCIOLOGIA

Tabla P8 bis.

Eficiencia instituciones

\begin{tabular}{lrrrrrrr}
\hline & Ayto & $\begin{array}{c}\text { Mancom. } \\
\text { Municipios }\end{array}$ & Diputación & GDR & IFA & $\begin{array}{r}\text { Junta } \\
\text { Andalucía }\end{array}$ & $\begin{array}{c}\text { Entidades } \\
\text { Bancarias }\end{array}$ \\
\hline Ninguna & & & & & & & \\
eficiencia & $7,0 \%$ & $7,6 \%$ & $6,5 \%$ & $3,4 \%$ & $6,5 \%$ & $4,5 \%$ & $11,5 \%$ \\
2 & $18,8 \%$ & $17,7 \%$ & $20,8 \%$ & $5,6 \%$ & $19,9 \%$ & $18,5 \%$ & $20,5 \%$ \\
3 & $32,0 \%$ & $26,7 \%$ & $39,6 \%$ & $21,3 \%$ & $31,7 \%$ & $37,1 \%$ & $27,2 \%$ \\
4 & $25,3 \%$ & $16,0 \%$ & $17,4 \%$ & $30,3 \%$ & $16,6 \%$ & $18,5 \%$ & $21,9 \%$ \\
Mucha & & & & & & & \\
eficiencia & $13,8 \%$ & $7,9 \%$ & $5,1 \%$ & $24,2 \%$ & $7,0 \%$ & $9,0 \%$ & $11,0 \%$ \\
NS/NC & $3,1 \%$ & $24,2 \%$ & $10,7 \%$ & $15,2 \%$ & $18,3 \%$ & $12,4 \%$ & $7,9 \%$ \\
TOTALES & 356 & 356 & 356 & 356 & 356 & 356 & 356 \\
& $100 \%$ & $100 \%$ & $100 \%$ & $100 \%$ & $100 \%$ & $100 \%$ & $100 \%$ \\
Media & 3,21 & 2,99 & 2,93 & 3,78 & 2,97 & 3,10 & 3,00 \\
Desv. típica & 1,13 & 1,12 & 0,97 & 1,06 & 1,05 & 1,02 & 1,20 \\
\hline
\end{tabular}

